\title{
Disability design and innovation in computing research in low resource settings
}

Dafne Zuleima Morgado-Ramirez ${ }^{1,2}$, Giulia Barbareschi ${ }^{1,2}$, Maggie Donovan-Hall ${ }^{3}$, Mohammad Sobuh $^{8}$, Nida' Elayan ${ }^{8}$, Brenda Nakandi ${ }^{9}$, Robert Ssekitoleko ${ }^{9}$, Joyce Olenja ${ }^{4}$, Grace Magomere $^{4}$, Sibylle Daymond ${ }^{5}$, Jake Honeywill ${ }^{5}$, Ian Harris ${ }^{5}$, Nancy Mbugua ${ }^{6}$, Laurence Kenney ${ }^{7}$, Catherine Holloway ${ }^{1,2}$

1 University College London, UK; 2 Global Disability Innovation Hub, UK; 3 University of Southampton, UK; 4 University of Nairobi, Kenya; 5 Motivation UK; 6 Motivation Africa; 7 University of Salford, UK; 8 University of Jordan, Jordan; 9 Makerere University, Uganda

\begin{abstract}
$80 \%$ of people with disabilities worldwide live in low resourced settings, rural areas, informal settlements and in multidimensional poverty. ICT4D leverages technological innovations to deliver programs for international development. But very few do so with a focus on and involving people with disabilities in low resource settings. Also, most studies largely focus on publishing the results of the research with a focus on the positive stories and not the learnings and recommendations regarding research processes. In short, researchers rarely examine what was challenging in the process of collaboration. We present reflections from the field across four studies. Our contributions are: (1) an overview of past work in computing with a focus on disability in low resource settings and (2) learnings and recommendations from four collaborative projects in Uganda, Jordan and Kenya over the last two years, that are relevant for future HCI studies in low resource settings with communities with disabilities. We do this through a lens of Disability Interaction and ICT4D.
\end{abstract}

\section{CCS CONCEPTS}

- Human-centered computing $\sim$ Human computer interaction $(\mathrm{HCI}) \sim \mathrm{HCI}$ design and evaluation methods $~$ Field studies $\bullet$ Social and professional topics $\sim$ User characteristics $\sim$ People with disabilities

\section{KEYWORDS}

Disability Innovation, ICT4D, Inclusive Design, Disability, Low resource settings, Disability Interaction

\section{Introduction}

Over $15 \%$ of the global population reports some type of disability, which in most countries represents the largest single minority group [1-3]. Furthermore, approximately $80 \%$ of people with disabilities (PWD) worldwide live in low resourced settings e.g. rural areas and informal settlements, which result in multidimensional poverty [4-7]. HCI research has shown how technology, can be a valuable tool that increases independence, empowers PWD and enables better access to opportunities [8-11]. Similarly, numerous ICT4D, international development researchers and practitioners have leveraged the power of technological innovations to deliver programs that aim to increase opportunities and improve the lives of people living in low resourced settings [12-14]. Various studies have started to investigate the possibilities for technological innovations to empower and support PWD who live in low resourced settings [15-33].

Our work as HCI, disability and assistive technology researchers in low resourced settings is guided by the "Disability Interaction (DIX)" manifesto [34], a challenge-based approach to create a more inclusive world where disability can be an inspiration for innovation and creativity, and where inclusion is the norm. Over the years we came to conceptualize DIX as a novel way of thinking, driven by principles of openness to novel interactions and bottom-up approaches to participatory innovation; with the aim to empower PWD and unlock opportunities, especially where these opportunities are scarce to begin with. DIX places PWD at the core of all our research: starting from listening to peoples' lived experiences, creating partnerships that can harness the creativity of the disabled community and co-creating non-binary solutions that align with the values of 
different environmental, social and cultural contexts [34]. DIX expands on approaches such as Ability-based design[35]. DIX seeks to solve global problems and leveraging not only technology innovations but the full ecosystem of disability innovation including policy and practice.

Within the context of our international development work in low resourced settings we strive for a postcolonial approach [13,36,37] where culture is seen as a dynamic construct rather than a fixed geographical attribute, goals are shaped according to the aspirations of the communities we work with; rather than pushing solutions based on a pre-determined political agenda. We adapt research methods to preserve the societal voice that shapes the knowledge we collect as researchers. We know technology can deliver significant societal benefits, but when parachuted in without awareness or regard, , it amplifies societal gaps [38-41].

These commitments often mean that research delivery is as important as the results that are produced as part of our academic outputs. This is not just referring to the methods used but also, the partnerships that are developed, the strategies for communication implemented by researchers, the ethics processes that are managed within the research, the logistic challenges associated with international funding and other practical implications that determine the success or failure of a project. We assume that these challenges are shared by many other researchers in the field. Nonetheless, most ICT4D publications involving PWD, largely focus on the results of the research carried out rather than on the process of developing and delivering it.

The aim of this experience report is twofold: (1) to give an overview of the work HCI work that has been done with a focus on disability and in low resource settings, and (2) to share our journey of thinking and reflections from four collaborative projects in Uganda, Jordan and Kenya over the last two years. We do this through a lens of DIX, expanding the thinking for this emerging sub-domain of HCI to better include ICT4D thinking.

\section{Related Work}

The ACM Guide to computing literature database was used to search for past work related to computing research in low resource settings and in relation to disabled communities (June 2020). Key terms related to low resource settings and disability were used. No time limit was set. 46 relevant references were identified. We present a brief review of 19 included references. Proceedings and research articles that did not perform research in relation to disability and that did not include members of disabled communities were excluded.

Most of the studies we found focused on understanding technology currently in use or develop new technologies for PWDs in low resourced settings. Technologies used or studied are interactive voice response [15, 25, 29, 32], wheelchair transporter [16], assistive technology as a system [17], computer based [18, 27, 31], screen readers [19], online social networks [20], mobile application [21], audio content [22], mobile phones [23, 26, 33] and gesture-based technology [24, 28]. From 2008 up to 2020 there has been a steady and dominant production of disability and HCI research in low resource setting with a focus on visual impairments ( 8 out of 19 studies and 2 other studies with a focus on various disabilities including visual impairment) [15, $20-22,25,26,29,33]$. The rest of the studies focused on mobility impairments [16, 19], mixed visual and mobility impairments [23, 32], mixed developmental and intellectual disabilities [24, 28], mixed unspecified disabilities [17, 18], intellectual disability [28], developmental disability [31] and hearing impairment [32].

The geographical locations of disabled communities that have been involved in the identified studies are: India [23, 25, 28, 32], Brazil [19, 20, 27], China [18, 21], Pakistan [17, 29], South Africa [15], Sierra Leone [23, 26], Namibia [31], Kenya [33], Malawi [26], Rwanda [26], Costa Rica [26], Cambodia [16] and Turkey [30]. When reviewing the selected corpus, we searched for descriptions of challenges and learnings experienced by the researchers and their collaborators when performing HCI and disability innovation in low resource settings. Eight studies did not report challenges and learnings related to HCI and disability research in low resource settings [16-21, 29, 30,33]. Studies that reported learnings that could translate to other HCI and disability studies in low resource settings, focused on: institutional cultures and processes[18], multidimensional complexity of access to ICT [23], high expectations of participants and low technology acceptance [24], understanding that technology alone cannot solve multidimensional problems [25, 26, 32], that participant recruitment is difficult when a specific disability is an inclusion criteria [26] and that sometimes PWD do not need the intervention of researchers in order to adopt technology [22].

Only eight studies gave recommendations that would be useful for future computing researchers wanting to perform DIX studies in low resource settings. A common theme among the recommendations was to utilize 
in-depth qualitative data and participatory and used-centred research while taking into account local social, cultural and economic dimensions, ICT access and inequalities of the context in which PWD live [19, 23, 26] [28]. Also that to benefit PWD that need assistive technology, it is necessary to bring manufacturers, promoters and end users together to come up with social corporate modalities that satisfy all [16]. Finally, enable civic engagement, promote demands for change and meaningful action and support empowerment of PWDs [30]. Despite providing these overarching recommendations none of the studies focused specifically on the challenges encountered and the lessons learned in delivering their research including aspects such as gaining ethics approval, building partnerships with local organizations and sustaining engagement with participants.

We found limitations shared across the majority of the identified studies: not reporting ethical approval and data protection, not acknowledging participants and/or other stakeholders and not including or seeking the "voice" of disabled end users for the design phase and/or at any point of the study.

\section{Four disability innovation studies in low resource settings}

We present four studies from three low resourced settings (Uganda, Jordan and Kenya) that, while having different goals, they share the aim of disability innovation, challenges, learnings. The studies in Uganda and Jordan are part of a project involving four UK universities and universities in Uganda and Jordan that started with scoping work to inform the design of the main studies and involved UK visiting Uganda and Jordan for one week [42]. The studies in Kenya were conducted as part of the AT2030 - Life Changing Assistive Technology for All program [43], funded by the UK Department for International Development and led by the Global Disability Innovation Hub. Some of the partnerships that enabled the delivery of these projects had been set up as part of AT2030, with some organizations being involved in multiple projects, whereas others participated only in individual projects.

\subsection{Real life use of upper limb prosthetics}

This study explored how prosthetics are used in real life by people with upper limb absence and how individuals managed if they did not use a prosthesis. Some of the partners had worked together previously, and the team was guided by an experienced advisory board with representatives from major NGOs. We utilized wearable wrist worn activity monitors, an activity monitoring diary, feature phones to collect experience diaries and end of day surveys. We encouraged participants with and without a prosthesis to create experience diaries through feature phones that we provided or with their own. Mobiles enabled the diaries to be captured as audio, video and photos, in order to suit all levels of literacy and spoken language.

Specific Challenges. contractual delays of up to 6 months delayed hiring of research staff in Uganda and Jordan. A delay of this length had not been factored into either the budget, or work plan. Although a co-design approach was utilized, while leveraging the HCI expertise of the UK team. However, feedback from collaborating institution was slow and required multiple meetings over a year. This delayed data collection until the initial methods were adapted through a process of consultation. Cultural adaptations were adopted, e.g. participants from Jordan did not complete experience and activity monitoring diaries and instead completed quick surveys while participants in Uganda created a significant amount of audio, videos, and photos as part of the experience and activity monitoring diaries. In reflection, the team agreed that had the teams been introduced physically together to understand language, ways of working and expertise brought by each individual, they would have felt comfortable in raising questions and concerns sooner than later.

\subsection{Support network of people with upper limb absence}

This study aimed to understand the communication and support needs of individuals with upper limb absence interested in meeting other people with similar disabilities; while testing if removing the barrier to access mobile phones and mobile data enabled the development of such support networks. UK HCI researchers proposed the structured creation of an informal support network that would be managed and maintained by individuals with upper limb absence without moderation or any type of intervention from part of the researchers. We only asked participants to complete an experience diary in their method of preference (written and/or with a mobile phone to record audio, video or photos). 
Specific challenges. Despite applying changes to the protocol to attempt to suit cultural norms in Jordan (for example, structuring two groups by gender and organizing a workshop to introduce each other in person before starting the remote network), participants did not engage with this study. People chose not to call or text or complete the experience diary despite having previously indicated interest in talking and establishing relationships with other people with upper limb absence during the scoping study, this did not happen in practice. This study did not take place in Uganda due to concerns that potential participants would misconstrue taking part in the study as akin to the establishment of a formal Ugandan Association of Upper Limb Amputees. It was felt that running the study this could lead to disappointment and potential harm.

\subsection{Mobile phones use in informal settlements}

To build a better understanding of how people with different disabilities who live in informal settlements interact with their mobile phones in their daily lives, we organized four studies that engage people with visual, mobility, hearing and learning impairments, respectively. To date, only the first two studies involving people with visual and mobility impairment have been conducted [33, 44]. Both studies involved semi-structured interviews and ethnographic observations. We have completed a co-design workshop to explore visually impaired participants' aspirations for more inclusive future technologies.

Specific challenges. NGOs working in the disability sector are often "impairment specific". We therefore had to take time forging new partnerships with dedicated NGO's and train a new group of community researchers. This increased the expenses. Furthermore, it was difficult to strike a balance between informing participants of their legal rights without overwhelming them with information. Some participants had limited literacy, others found reading information in braille tiring and time consuming, some skipped sections of the information sheet or asked to be given a short summary of it. We wanted to ensure that participants were rightfully reimbursed for any expense, yet local partners advised against any payment beyond $20 \$$ for a day of participation in research activities as greater sums could be coercive and possibly put them at risk of robbery. We were unable to conduct a co-design workshop that we had planned with participants with a mobility impairment, due to logistic and accessibility challenges which limited the impact of our research.

\subsection{Leveraging 3D printing technology for wheelchair provision}

The aim of this study was to test the feasibility and acceptability of an innovative model of wheelchair provision that leverages the use of Computer Aided Design parametric modelling and 3D printing to manufacture bespoke wheelchairs for Kenyan users. Local wheelchair providers were trained for two months in the new tools and methods for wheelchair assessment, production and fitting. Then, for three months, local providers independently produced wheelchairs for six local expert users, who evaluated the wheelchairs. We conducted repeated observations and interviews respectively during and after each section of training and independent delivery. More information on the outcomes of the project are published elsewhere [45].

Specific challenges. Finding an appropriate site for the project and establishing the initial partnership with a local organization that provides wheelchairs to local users required a significant amount of time and several adjustments to the initial research protocol and timeline. Although many small and medium sized organizations in Kenya occasionally provide wheelchairs to local people with disabilities, few follow the appropriate guidelines established by the WHO [46]. As our study sought to trial a new model for provision of bespoke wheelchairs in a timeframe that was relatively short. This significantly restricted our initial choice for a local partner. Furthermore, the project required local staff to undertake several hours of training a week for the first stage of the project. This had to be negotiated with management to minimize disruption to the regular provision of services to the local community. Finally, managing the expectation of local users to understand that they could not keep the wheelchairs was challenging. Some users would have preferred to keep the new wheelchairs to use them as a backup or as a more attractive alternative for "special occasions".

\section{Lessons learned and discussion}

This section takes a reflective approach to consider the lessons we feel we have learnt across the studies. Initial thoughts were shared as a team to provide a 'collaborative reflective consideration' and ensure that the issues 
identified were considered from different perspectives and viewpoints. This is part of our DIX ethos outlined in the introduction section and our overall commitment to building partnerships and learning from each other. We have purposely provided limited details as specific situations and experiences to maintain anonymity as it is the issue being discussed that is important and not necessarily the specific context.

\subsection{Scoping study or patient and public involvement}

We promote the importance of thorough scoping and exploratory work. As well as providing valuable insights for the study design and possible technological solutions, these activities were fundamental for developing working relationships within and beyond the research team, understanding training or capacity building needs across countries, identifying potential partners, building relationships with organizations, identifying local suppliers (in terms of equipment, space or local technical support), understanding timings and processes involved in contractual agreements being finalized, shipping materials between countries, and evaluating the appropriateness of the available infrastructure. Scoping work involved a series of observation visits to a wide range of stakeholders (i.e. departments in public hospitals, non-government organizations, private clinics and industrial companies) and informal discussion with people with disabilities and their families. We recommend that scoping trips are carried out by a multidisciplinary team and, while they incorporate pre-scheduled visits, they allow for flexibility to capture opportunities, visit unexpected locations and meet potential volunteers. Making a report from the scoping study that can be shared with the wider team of the research project is a useful way of communicating the learnings and share details of the context using different media.

\subsection{End users voice}

Researchers working in diverse contexts should acknowledge their own biases but also be mindful of the cultural biases that surround the research team, which might affect participant engagement. Guides, community leaders with disabilities and local researchers are not just a key to access participants. They are valuable allies that can help to define and evaluate research approaches to ensure they are culturally appropriate and support data analysis to prevent foreign researchers from altering participants' voices or extrapolating messages out of their context [13]. An effective strategy is to establish a steering group led and selected by local PWDs, to help define and review the research plan and check the suitability and appropriateness of the language and methods. Feedback from end users could also be valuable in enabling researchers to modify methods and approaches throughout data collection periods. We recommend having frequent communication with the participants and intervene by modifying the methods in order to prevent harm to participants and the research teams (i.e. the community stigmatizes them for participating in research).

\subsection{Understanding different expectations}

Expectations will differ between institutions, organizations, processes, values, disciplines, cultures, ways of working, people management and access to ICT. Leading researchers shall debrief the team about these likely misalignments while suggesting ways of managing them, we recommend a risk assessment. We reflect on managing expectations across working groups within the research team. In one study that involves the development of a new device, it was a challenge to provide a list of design requirements at speed that the engineering team due to the length of time required to thoroughly analyze qualitative data, We have managed this by reiterating the importance of including the voice of end users. Yet, methods of communicating interim findings from the user-led studies need to be found and integrated into the design of the qualitative work.

With researchers from different countries there can be tensions between wanting to co-design research and leveraging leadership from more experience researchers. If not properly managed, these tensions can feel disempowering and potentially damage the partnership that is essential for the success of the project. Our experience was that all researchers had complimentary expertise that was crucial to the growth of the team. Researchers from low-resourced settings were often unfamiliar with HCI methods and they view positively the idea of learning from experienced researchers from experienced settings. At the same time, foreign researchers were keen to learn from the first-hand experience of local researchers to understand how traditional research approaches could be adapted to the local context. These expectations need to be discussed with candor 
at the pre-funding or start of the project. Other relevant expectation that was mismatched is the access to ICT necessary for work and communication (internet, working laptops, remote collaboration software that is not free, etc.). Disparity of ICT access also led to challenges in ensuring appropriate acknowledgement of contribution for community researchers, where authorship registration for publications requires institutional affiliations and email addresses to which they rarely have access.

Expectations can also be complex to manage in the relationship with participants. Formalized processes such as risk table to guide researchers' responses can help. However, in one of our projects some colleagues did not engage with this process and sought ad hoc support via email to address individual situations. In some cases, participants' expectations went beyond what our research could deliver and had to continually be adjusted. Although information sheets were co-drafted and participants debriefed, we repeatedly had to clarify that, as part of the research we could not provide free assistive devices, mobile phones or financial support.

\subsection{Communication and relationships}

Building relationships across teams takes time and, in some cases, it required one year to establish a fluid working relationship. Communication is crucial and a flexible trial and error process is required to find tools and strategies that work for all stakeholders. Communication is a two-sided process and both sides need to make compromises and invest time in learning about the other side's culture and ways of working. For instance, the working days are different in some countries (Sunday to Thursday). alongside other responsibilities. When working remotely it might be necessary to utilize various interfaces for communication and collaboration as some do not work as well in all contexts and colleagues will not always have access. However, transitions between interfaces should be carried out in an organized manner to prevent confusion. Having sparse remote work-related meetings at the beginning of the project might not be enough to develop a strong relationship, organize regular informal meetings or other activities that can help to build a rapport for better communication. During the delivery of our studies, we found helpful to have shorter weekly meetings, in addition to longer established research management monthly meetings. However, stakeholders agreed that long and remote meetings are difficult to follow, tiring and not very useful. For projects that span multiple months, it is important for the team to be able to also engage with each other in person at regular intervals, to maximize the relationship and address challenges that arise during the research. As relationships become stronger overtime this need might reduce but when establishing budgets for international projects, the need for in-person engagement between researchers should be considered. Finally, communication and accessibility needs of stakeholders who have different first languages and abilities should be considered.

\subsection{Ethics}

Data protection and ethical approval to perform the study will ideally have been sought and granted from all institutions involved. Ethics committees' guidelines and data protection legislations are articulated differently amongst countries and institutions. This could cause potential discrepancies between requirements from ethics and data protection boards of different countries. We suggest using the criteria that offers utmost protection to participants and follows the best participant informed consent procedures. Some of our colleagues preferred that UK researchers sought ethics approval first. As processing times might vary, we also recommend scoping the typical time for ethics approval at all institutions involved. Application processes differ significantly, and, in some instances digital applications are not accepted and stakeholders need to visit in person a variety of staff in a hierarchical custom (time consuming and tiring for the stakeholders). Many smaller organizations in low-resourced settings might not have access to their own review boards for ethics application. Finally, when disseminating the results through publications and community engagement it is important to mention that the research was reviewed by an ethics committee and that the data of participants is protected.

\subsection{Funding}

Funding was obtained in the UK for all the four presented and thus had to be transferred to the collaborating institutions in other countries. This process was more difficult and took longer than expected. We recommend scoping: the timeline and processes of drafting contracts, transferring and accessing funds at all stakeholders' 
institutions. In some countries, even when the funding has been successfully transferred, the local process of hiring a research assistant will be complex and prolonged. Finally, when funding is delayed, some funding partners recommend spending personal finances and asking for reimbursements. For some, this expectation is impossible, any expenditure without funding on site is not reimbursed. All the above understanding takes time, becomes visible in multiple meetings and highlights the importance of flexible approaches.

\subsection{Reflections on remote data collection and analysis}

We recommend: 1) field researcher keep a research diary with two components: research related notes and personal reflections, 2) agree on a system to organize data as it is captured; 3) utilize digital interfaces to coanalyze anonymized data. In some studies, depending on the funding agreement, it is possible to hire an experienced local company to collect data. Finding, selecting and debriefing the provider requires time and resources and should be scheduled for in the research plan. Finally, when experienced researchers are not available in a country, hiring more junior members and working closely with them will help to build sustainability [47]. Routine meetings with local experienced staff are essential for both training and mentoring, and international experience of co-authorship of publications should be encouraged.

\section{Conclusion}

To conclude, we present a visual representation of elements and dimensions that will help HCI researchers plan future research with a DIX lens and in low resource settings (Figure 1). There are five temporal stages of the project: pre-funding, start-up, delivery, wind-down and post-funding. And for each stage there are six dimensions: communication and relationships, co-designed research plan, funding, ethics and data protection, human resources and sustainability. Across dimensions and temporal stages, we are proposing components that together draw the most positive expected scenario for conducting a DIX study in low resource settings. The length of the horizontal arrows indicates approximate duration of components, with a start edge and an undefined duration, that will be different for each project. Key components in the dimension of communication and relationships are the presential "rapport meetings" that we recommend based on our learnings.
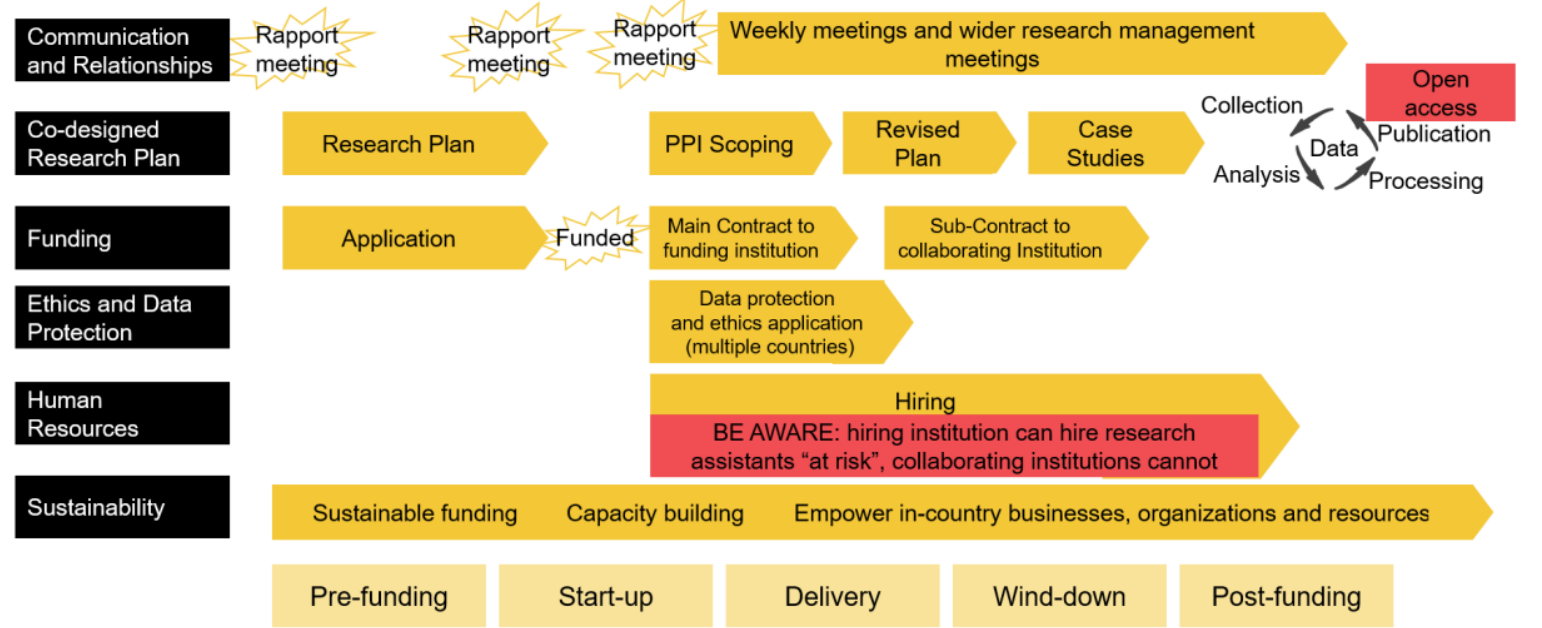

Figure 1: Most positive expected scenario for conducting a DIX study in low resource settings. Elements and dimensions that will help HCI researchers plan future research with a DIX lens and in low resource settings.

\section{ACKNOWLEDGMENTS}

We thank the participants of all the studies mentioned here, UKAID (AT2030 Programme 201879-108), UK Global Challenges Research Fund through the Engineering and Physical Sciences Research Council and National Institute for Health Research (EP/R013985/1) for funding this research. 


\section{REFERENCES}

1. Kostanjsek, N., et al., Counting disability: global and national estimation. Disability and Rehabilitation, 2013. 35(13): p. 1065-1069.

2. $\quad$ World Health Organization, World Report on Disability. 2011.

3. Lazar, J. and P. Jaeger Reducing Barriers to Online Access for People with Disabilities. Issues in Science and Technology, 2011. 27.

4. Grech, S., Disability and Poverty: Complex Interactions and Critical Reframings, in Disability in the Global South: The Critical Handbook, S. Grech and K. Soldatic, Editors. 2016, Springer International Publishing: Cham. p. 217-235.

5. Grech, S., Disability and Development: Critical Connections, Gaps and Contradictions, in Disability in the Global South: The Critical Handbook, S. Grech and K. Soldatic, Editors. 2016, Springer International Publishing: Cham. p. 3-19.

6. Pinilla-Roncancio, M., et al., Multidimensional poverty and disability: A case control study in India, Cameroon, and Guatemala. SSM - Population Health, 2020. 11: p. 100591.

7. Robles Aguilar, G. and A. Sumner, Who are the world's poor? A new profile of global multidimensional poverty. World Development, 2020. 126: p. 104716.

8. Kane, S.K., et al., Freedom to roam: a study of mobile device adoption and accessibility for people with visual and motor disabilities, in Proceedings of the 11th international ACM SIGACCESS conference on Computers and accessibility. 2009, Association for Computing Machinery: Pittsburgh, Pennsylvania, USA. p. 115-122.

9. Gallagher, B. and H. Petrie, Initial results from a critical review of research on technology for older and disabled people, in Proceedings of the 15th International ACM SIGACCESS Conference on Computers and Accessibility. 2013, Association for Computing Machinery: Bellevue, Washington. p. Article 53.

10. Branham, S.M. and S.K. Kane, Collaborative Accessibility: How Blind and Sighted Companions Co-Create Accessible Home Spaces, in Proceedings of the 33rd Annual ACM Conference on Human Factors in Computing Systems. 2015, Association for Computing Machinery: Seoul, Republic of Korea. p. 2373-2382.

11. Rajapakse, R., M. Brereton, and L. Sitbon, Design Artefacts to Support People with a Disability to Build Personal Infrastructures, in Proceedings of the 2018 Designing Interactive Systems Conference. 2018, Association for Computing Machinery: Hong Kong, China. p. 277-288.

12. Heeks, R., ICT4D 2.0: The Next Phase of Applying ICT for International Development. Computer, 2008. 41(6): p. 26-33.

13. Irani, L., et al., Postcolonial computing: a lens on design and development, in Proceedings of the SIGCHI Conference on Human Factors in Computing Systems. 2010, Association for Computing Machinery: Atlanta, Georgia, USA. p. 1311-1320.

14. Gupta, A., et al., mClerk: enabling mobile crowdsourcing in developing regions, in Proceedings of the SIGCHI Conference on Human Factors in Computing Systems. 2012, Association for Computing Machinery: Austin, Texas, USA. p. 1843-1852.

15. Greeff, M., L. Coetzee, and M. Pistorius, Usability evaluation of the South African National Accessibility Portal interactive voice response system, in Proceedings of the 2008 annual research conference of the South African Institute of Computer Scientists and Information Technologists on IT research in developing countries: riding the wave of technology. 2008, Association for Computing Machinery: Wilderness, South Africa. p. 76-85.

16. Owens, J. and C. Simonds, Beyond the wheelchair: development of motorized transport for people with severe mobility impairments in developing countries, in Proceedings of the 2nd International Convention on Rehabilitation Engineering \& Assistive Technology. 2008, Singapore Therapeutic, Assistive \& Rehabilitative Technologies (START) Centre: Bangkok, Thailand. p. 211-214.

17. Ahmad, M. and M. Morshed Ahmad, Who should pay the price: state or disabled rural individuals? a low-income countries' perspective, in Proceedings of the 4th International Convention on Rehabilitation Engineering \& Assistive Technology. 2010, Singapore Therapeutic, Assistive \& Rehabilitative Technologies (START) Centre: Shanghai, China. p. Article 66.

18. Liu, Y. and J. Bacon, Innovation or diffusion? three scenarios of vocational education settings in ICT practices for people with learning difficulties in China, in Proceedings of the 4th International Convention on Rehabilitation Engineering \& Assistive Technology. 2010, Singapore Therapeutic, Assistive \& Rehabilitative Technologies (START) Centre: Shanghai, China. p. Article 8. 
19. Schibelsky G. Piccolo, L., E. M. De Menezes, and B. De Campos Buccolo, Developing an accessible interaction model for touch screen mobile devices: preliminary results, in Proceedings of the 10th Brazilian Symposium on Human Factors in Computing Systems and the 5th Latin American Conference on Human-Computer Interaction. 2011, Brazilian Computer Society: Porto de Galinhas, Pernambuco, Brazil. p. 222-226.

20. Magalhães, C.V.C., et al., Developing a social network of support to health care: the experience of GenNet, in Proceedings of the 18th Brazilian symposium on Multimedia and the web. 2012, Association for Computing Machinery: São Paulo/SP, Brazil. p. 351-354.

21. Shangguan, L., et al., CrossNavi: enabling real-time crossroad navigation for the blind with commodity phones, in Proceedings of the 2014 ACM International Joint Conference on Pervasive and Ubiquitous Computing. 2014, Association for Computing Machinery: Seattle, Washington. p. 787-798.

22. Vashistha, A., et al., Educational Content Creation and Sharing by Low-Income Visually Impaired People in India, in Proceedings of the Fifth ACM Symposium on Computing for Development. 2014, Association for Computing Machinery: San Jose, California, USA. p. 63-72.

23. Jones, J. and J. Pal, Counteracting dampeners: understanding technology-amplified capabilities of people with disabilities in Sierra Leone, in Proceedings of the Seventh International Conference on Information and Communication Technologies and Development. 2015, Association for Computing Machinery: Singapore, Singapore. p. Article 6.

24. Sharma, S., Designing Novel Gesture-based Services for Under Served Indian Communities, in Proceedings of the 7th International Conference on HCI, IndiaHCI 2015. 2015, Association for Computing Machinery: Guwahati, India. p. 161-164.

25. Vashistha, A., et al., Sangeet Swara: A Community-Moderated Voice Forum in Rural India, in Proceedings of the 33rd Annual ACM Conference on Human Factors in Computing Systems. 2015, Association for Computing Machinery: Seoul, Republic of Korea. p. 417-426.

26. Pal, J., et al., An Accessibility Infrastructure for the Global South, in Proceedings of the Eighth International Conference on Information and Communication Technologies and Development. 2016, Association for Computing Machinery: Ann Arbor, MI, USA. p. Article 24.

27. Figueredo de Santana, V., R. Laiola Guimarães, and A. Britto Mattos, Identifying challenges and opportunities in computer-based vocational training for low-income communities of people with intellectual disabilities, in Proceedings of the 13th Web for All Conference. 2016, Association for Computing Machinery: Montreal, Canada. p. Article 2.

28. Sharma, S., et al., Gesture-based Interaction for Individuals with Developmental Disabilities in India, in Proceedings of the 18th International ACM SIGACCESS Conference on Computers and Accessibility. 2016, Association for Computing Machinery: Reno, Nevada, USA. p. 61-70.

29. Raza, A.A., et al., Baang: A Viral Speech-based Social Platform for Under-Connected Populations, in Proceedings of the 2018 CHI Conference on Human Factors in Computing Systems. 2018, Association for Computing Machinery: Montreal QC, Canada. p. Paper 643.

30. Yildiz, Z., et al., Examining Online Practices of an Autism Parent Community in Turkey: Goals, Needs, and Opportunities, in Proceedings of the 2019 on Designing Interactive Systems Conference. 2019, Association for Computing Machinery: San Diego, CA, USA. p. 373-384.

31. Abiatal, L.K.S. and G.R. Howard, Constructivist Assistive Technology in a Mathematics Classroom for the Deaf: Going Digital at a Rural Namibian Primary School, in Proceedings of the South African Institute of Computer Scientists and Information Technologists 2019. 2019, Association for Computing Machinery: Skukuza, South Africa. p. Article 27.

32. Dubey, K., et al., Learnings from deploying a voice-based social platform for people with disability, in Proceedings of the 2nd ACM SIGCAS Conference on Computing and Sustainable Societies. 2019, Association for Computing Machinery: Accra, Ghana. p. 111-121.

33. Barbareschi, G., et al., The Social Network: How People with Visual Impairment use Mobile Phones in Kibera, Kenya, in Proceedings of the 2020 CHI Conference on Human Factors in Computing Systems. 2020, Association for Computing Machinery: Honolulu, HI, USA. p. 1-15.

34. Holloway, C., Disability interaction (DIX): a manifesto. interactions, 2019. 26(2): p. 44-49.

35. Wobbrock, J.O., et al., Ability-Based Design: Concept, Principles and Examples. ACM Trans. Access. Comput., 2011. 3(3): p. Article 9.

36. Sultana, S. and S.I. Ahmed, Witchcraft and HCI: Morality, Modernity, and Postcolonial Computing in Rural Bangladesh, in Proceedings of the 2019 CHI Conference on Human Factors in 
Computing Systems. 2019, Association for Computing Machinery: Glasgow, Scotland Uk. p. Paper 356.

37. Ahmed, S.I., N.J. Mim, and S.J. Jackson, Residual Mobilities: Infrastructural Displacement and Post-Colonial Computing in Bangladesh, in Proceedings of the 33rd Annual ACM Conference on Human Factors in Computing Systems. 2015, Association for Computing Machinery: Seoul, Republic of Korea. p. 437-446.

38. Toyama, K., Technology as amplifier in international development, in Proceedings of the 2011 iConference. 2011, Association for Computing Machinery: Seattle, Washington, USA. p. $75-82$.

39. Toyama, K., Geek Heresy - Rescuing Social Change from the Cult of Technology. 2015, United States of America: PublicAffairs. 352.

40. Wyche, S., N. Simiyu, and M.E. Othieno, Mobile Phones as Amplifiers of Social Inequality among Rural Kenyan Women. ACM Trans. Comput.-Hum. Interact., 2016. 23(3): p. Article 14.

41. Chew, H.E., I.P. Vigneswara, and M.R. Levy, When there's a will, there might be a way: the economic impact of mobile phones and entrepreneurial motivation on female-owned microenterprises, in Proceedings of the Sixth International Conference on Information and Communication Technologies and Development: Full Papers - Volume 1. 2013, Association for Computing Machinery: Cape Town, South Africa. p. 196-204.

42. Donovan-Hall, M., et al., Harnessing public and patient involvement for upper limb prosthetics design, in Trent International Prosthetics Symposium. 2019: Salford, United Kingdom.

43. Global Disability Innovation Hub. AT2030 Programme. 2020 [cited 202030 July]; Available from: https://at2030.org/.

44. Barbareschi, G., et al. Bridging the Divide: Exploring the use of digital and physical technology to aid mobility impaired people living in an informal settlement. in 22nd International ACM SIGACCESS Conference on Computers and Accessibility (ASSETS '20). 2020.

45. Barbareschi, G., et al. Value beyond function: Analyzing the perception of wheelchair innovations in Kenya. in 22nd International ACM SIGACCESS Conference on Computers and Accessibility (ASSETS '20). 2020.

46. World Health Organization, Guidelines on the provision of manual wheelchairs in less resourced settings. 2008: WHO Press. 124.

47. Kumar, N., et al., HCI Across Borders and Sustainable Development Goals, in Extended Abstracts of the 2020 CHI Conference on Human Factors in Computing Systems. 2020, Association for Computing Machinery: Honolulu, HI, USA. p. 1-8. 